La Revue

des Droits

de l'Homme

\section{La Revue des droits de l'homme}

Revue du Centre de recherches et d'études sur les droits fondamentaux

20 | 2021

Revue des droits de l'homme $-\mathrm{N}^{\circ} 20$

\title{
Quand le Conseil constitutionnel ne répond pas (vraiment) à la question. Les moyens irrecevables et les moyens inopérants dans les décisions QPC rendues par le Conseil constitutionnel
}

\section{Thomas Acar}

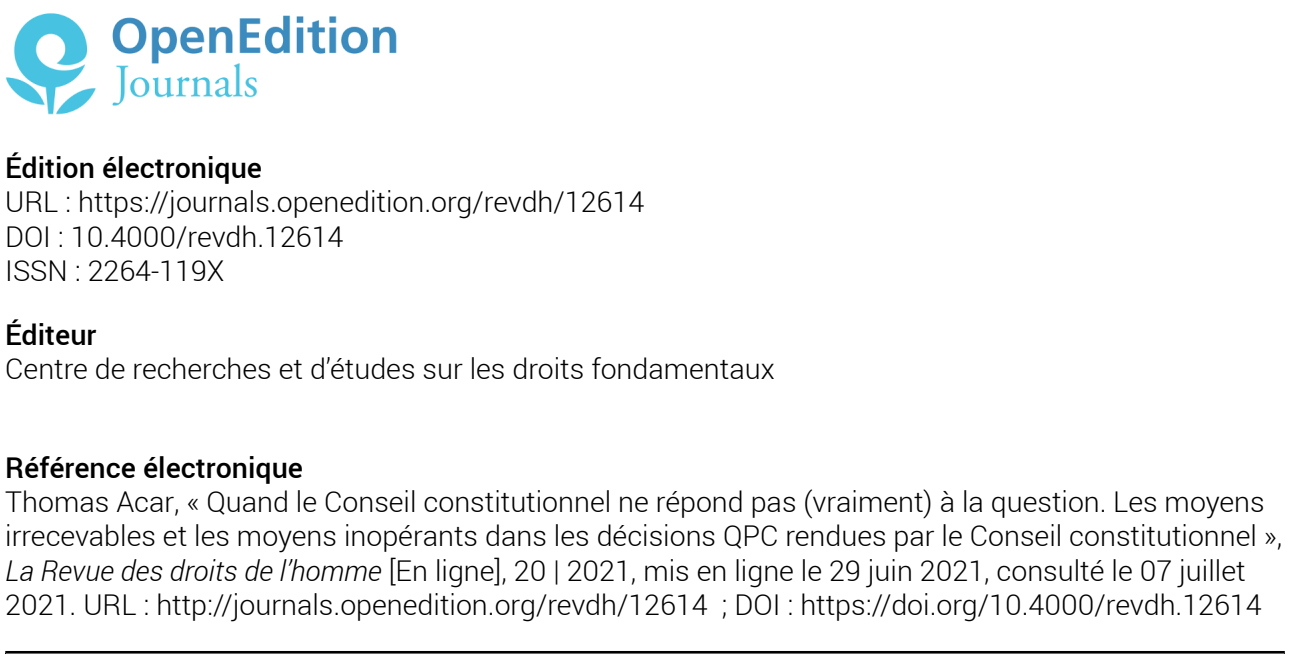

Ce document a été généré automatiquement le 7 juillet 2021.

Tous droits réservés 


\section{Quand le Conseil constitutionnel ne répond pas (vraiment) à la} question. Les moyens irrecevables et
les moyens inopérants dans les
décisions QPC rendues par le Conseil
constitutionnel

Thomas Acar

1 On attribue à Parménide l'aphorisme d'après lequel « l'être est, le non-être n'est pas » ; en découlerait une conception ontologique radicale concevant le mode d'existence des choses de manière absolument alternative et binaire : ou bien une chose est, ou bien elle n'est pas. S'oppose à cette perspective une conception ontologique plus modeste, attribuée à Héraclite, qui comprend les questions d'existence de manière plurielle, au prisme des contingences et des devenirs. Cette position s'incarne notamment dans l'idée célèbre suivant laquelle « on ne peut se baigner deux fois dans le même fleuve $»^{2}$.

2 L'étude des moyens irrecevables et inopérants dans le contentieux de la question prioritaire de constitutionnalité ne se satisfait guère d'une approche ontologique radicale et tranchée, et ce pour plusieurs raisons. Premièrement la distinction entre les moyens irrecevables et les moyens inopérants n'y est pas toujours chose aisée. Deuxièmement, la distinction entre ces moyens irrecevables ou inopérants et les moyens examinés au fond n'apparaît pas, dans certains cas, avec une netteté cristalline. Troisièmement, lorsqu'il apparaît possible d'identifier le caractère irrecevable ou inopérant d'un moyen, il va de soi que la logique même du contentieux de la question prioritaire de constitutionnalité ne confère à cette qualification qu'une pérennité relative, en ce que ce statut peut être appelé à évoluer au gré d'un changement de circonstances de fait ou de droit. Le contentieux qui nous occupe semble donc 
embrasser une approche plus sympathique de la conception relativiste de l'ionien au détriment de l'approche catégorique de l'éléate.

Avant d'expliquer plus précisément en quoi, il convient d'éclairer quelque peu l'objet de cette contribution en nous attachant à le définir. Si le syntagme «ne pas répondre à la question" emporte indubitablement un certain vague, d'une manière plus surprenante, les expressions «moyen irrecevable " et «moyen inopérant " apparaissent également souffrir d'une certaine indétermination, et ce même au sein du contentieux administratif qui les mobilise usuellement ${ }^{3}$.

Il semble de prime abord assez naturel de rapprocher le contentieux constitutionnel du contentieux administratif ${ }^{4}$. D'une part, ces deux contentieux entretiennent une fraternité historique, organique, et même géographique. D'autre part, les syntagmes « moyen irrecevable » et « moyen inopérant » apparaissent directement empruntés à la jurisprudence administrative et à la doctrine administrativiste.

Or, cette dernière définit le moyen inopérant comme le moyen "[insusceptible] d'exercer une influence sur la solution du litige $»^{5}$, tandis que le moyen irrecevable "est celui dont le juge est tenu, en l'état, de refuser d'apprécier le bien-fondé " ${ }^{6}$. Les deux catégories présentent donc un trait commun quant à leur conséquence - elles neutralisent l'appréciation du bien-fondé du moyen - tout en exhibant une différence de motivation nette pour y parvenir. Le moyen est inopérant en ce qu'il "ne peut conditionner l'issue de l'instance " ${ }^{7}$ alors que le moyen est irrecevable "lorsque le juge est tenu de l'évacuer précocement du débat ». On verra que cette distinction, en apparence claire, est en vérité problématique, tant à l'égard du contentieux administratif que du contentieux de la QPC qui nous occupe ici.

En réalisant cette recherche collective sur dix années de questions prioritaires de constitutionnalité, il nous est apparu qu'en dépit du double filtre opéré dans le cadre de la procédure QPC, par les juges du fond et par les juridictions suprêmes, toutes les questions renvoyées ne sont pas systématiquement et nécessairement examinées au fond par le Conseil constitutionnel. Tout au contraire, le Conseil s'arroge un rôle de troisième filtre qui le conduit parfois à ne pas répondre, partiellement ou totalement, à la question posée. Or, si une telle fonction de filtrage n'est pas explicitement prévue par les énoncés encadrant la procédure de la QPC ${ }^{9}$, elle semble cependant largement admise par la doctrine ${ }^{10}$, et s'impose en tout état de cause comme une réalité ${ }^{11}$. Il semble donc que se pose également, devant le Conseil, la question de la recevabilité et de l'opérance des moyens de constitutionnalité soulevés dans le cadre d'une QPC, préalablement à celle de leur bien-fondé. C'est cette motivation écartant les moyens par prétérition qui sera l'objet de notre démonstration: nous insisterons d'abord sur les difficultés qu'emporte l'objectivation de cette modalité du contrôle (I) avant de mettre en évidence les formes typiques que ces techniques peuvent prendre dans la motivation des décisions QPC (II).

\section{I- Les difficultés liées à l'objectivation du contrôle des moyens irrecevables et inopérants dans les décisions QPC}

7 Si l'on verra que dans le cadre du contentieux administratif «les théories de l'inopérance et de l'irrecevabilité des moyens peinent à être différenciées " ${ }^{12}$, il n'en va pas autrement dans 
le contentieux de la QPC. Bien au contraire, il semblerait que divers éléments conduisent à aggraver la confusion entre les moyens irrecevables et les moyens inopérants (A), quoiqu'il apparaisse in fine possible d'en proposer une distinction favorisant leur analyse respective (B).

\section{A. Éléments conduisant à une confusion pratique des moyens irrecevables et des moyens inopérants}

8 En principe, les moyens irrecevables et inopérants sont strictement distincts : le moyen irrecevable n'est pas nécessairement inopérant et réciproquement le moyen inopérant n'est pas nécessairement irrecevable. Toutefois le contentieux de la QPC pose un problème d'individuation net à cet égard puisque la motivation adoptée entretient ponctuellement une confusion certaine entre les moyen inopérants et les moyens inopérants. En outre, il apparaît périlleux de discerner strictement la recevabilité procédurale de la question dans son ensemble de la recevabilité des moyens qui la composent. En effet, les critères constitutifs de la QPC et plus particulièrement la nécessité, pour que la question soit recevable, de soutenir qu'une disposition législative porte atteinte aux droits et libertés que la Constitution garantit, suppose en elle-même une étude attentive de la recevabilité des moyens invoqués. Pour le dire simplement, en QPC la recevabilité de la question est très largement gouvernée par la recevabilité des moyens qui la constituent ${ }^{13}$.

9 Premièrement, au terme d'argumentations qui apparaissent formellement similaires le Conseil peut conclure ou bien à l'inopérance, ou bien à l'irrecevabilité d'un moyen. Il en va ainsi lorsque le Conseil constitutionnel identifie un moyen inopérant car la disposition législative à même de constituer une atteinte aux droits et libertés que la Constitution garantit ne lui est pas soumise ${ }^{14}$. Or, c'est en conclusion d'un raisonnement parallèle que la haute instance retient que lorsqu'il n'est pas saisi des dispositions législatives pertinentes, "il n'y a pas lieu [...] d'examiner l'argument tiré de ce qu'il en résulterait une atteinte inconstitutionnelle $»^{15}$. On en conclut alors qu'une motivation comparable, pour ne pas dire identique, conduit le Conseil à qualifier alternativement le moyen d'inopérant ou d'irrecevable, la terminologie du non-lieu à statuer marquant dans la jurisprudence relative à la QPC l'irrecevabilité des moyens ${ }^{16}$.

Deuxièmement, une difficulté surgit à l'occasion de l'examen de la capacité du moyen à démontrer une atteinte aux droits et libertés que la Constitution garantit ${ }^{17}$. Une telle capacité emporte un traitement différencié selon qu'elle est appréciée par les juridictions du filtre ou par le Conseil constitutionnel. Plus précisément, si les premières l'apprécient sous l'angle de la recevabilité du moyen, le second la traite au prisme de l'inopérance.

11 Cette condition expresse de l'article 61-1 C. n'est pas un critère explicite de la transmission de la question de la juridiction ordinaire à la juridiction suprême, ni un critère de renvoi de la question par cette dernière au Conseil constitutionnel, quoique la mention aux droits et libertés que la Constitution garantit apparaisse incidemment dans les dispositions relatives à la transmission et au renvoi ${ }^{18}$. Les juridictions ordinaires et les juridictions suprêmes tirent néanmoins un pouvoir d'appréciation large de la nécessité pour la question de ne pas être dépourvue de caractère sérieux ${ }^{19}$. En effet, le respect de ce critère est jugé à partir d'un examen approfondi non seulement des dispositions législatives contestées, mais également des normes 
constitutionnelles invoquées et de l'interprétation qu'a pu en faire le Conseil constitutionnel. Il donne en pratique lieu à un contrôle de constitutionnalité substantiel "décomplexé $\|^{20}$, notamment de la part des juridictions suprêmes ${ }^{21}$. L'étendue de ce contrôle se mesure par exemple au fait que le caractère sérieux n'est pas avéré non seulement lorsque la disposition contestée ne porte pas atteinte à un droit et liberté que la Constitution garantit, mais au-delà, lorsque l'atteinte portée n'est pas disproportionnée ${ }^{22}$. En dépit de son intensité, un tel contrôle se présente néanmoins sous les traits d'un contrôle de la recevabilité du moyen invoqué.

Cela n'est pas le cas devant le Conseil constitutionnel, qui mobilise plus volontiers l'instrument du " moyen inopérant » lorsqu'il est confronté à un moyen qui n'invoque pas dûment l'atteinte à un droit ou une liberté que la Constitution garantit ${ }^{23}$. On arguera à raison que le contrôle opéré alors diffère en nature de celui opéré par les juridictions du filtre ${ }^{24}$. Ces dernières apprécient le caractère sérieux de la question pour juger de la nécessité de sa transmission ou de son renvoi, tandis que le Conseil n'est nullement tenu à cet office et apprécie plus généralement la conformité de la disposition renvoyée aux droits et libertés que la Constitution garantie. Si ce point est indéniable, il n'en demeure pas moins que la dissonance entre le parallélisme des formes et la divergence des motivations pose un problème pratique de conceptualisation. Une motivation conduisant à déclarer un moyen irrecevable devant les juridictions du filtre peut se retrouver, sous une forme peu ou prou identique, pour justifier de l'inopérance d'un moyen devant le Conseil constitutionnel.

Troisièmement, la terminologie adoptée par la haute instance peut laisser planer un doute quant à la qualification du moyen. C'est notamment le cas des décisions de nonlieu à statuer. Cette terminologie est dédiée, dans le contentieux administratif, aux situations dans lesquelles «le juge estime ne plus avoir à intervenir dans le litige parce que cette intervention est devenue inutile en cours d'instance ou parce qu'une circonstance extérieure de fait ou de droit l'a rendue impuissante à en modifier les données $»^{25}$. Un tel dispositif apparaît dès lors consacrer "l'extinction du litige à raison de la disparition, en cours d'instance, de son objet ${ }^{26}$, il appartient à ce titre au registre des incidents de procédure. Ce cas de figure se présente ponctuellement devant le Conseil lorsque celuici «s'est prononcé sur la constitutionnalité d'une disposition législative postérieurement à l'enregistrement d'un mémoire soulevant une question identique $"{ }^{27}$. Dès lors, la haute instance ayant déjà répondu à la question, et l'existence d'un changement de circonstances dans ce bref délai étant hautement improbable, elle conclut logiquement au non-lieu à statuer ${ }^{28}$. Toutefois le Conseil constitutionnel fait une utilisation très large de ce dispositif puisqu'il considère non seulement n'y avoir pas lieu à statuer lorsqu'il s'est prononcé antérieurement au renvoi de la question par le Conseil d'état et la Cour de cassation et qu'il n'y a pas de changement de circonstances ${ }^{29}$, mais aussi lorsque la question excède sa compétence ou apparaît fondée sur des moyens irrecevables ${ }^{30}$. Le recours extensif au non-lieu à statuer et le sens spécifique que lui confère ainsi le contentieux de la QPC au regard de la signification usuelle qu'il a droit administratif s'explique largement par des considérations de diplomaties jurisprudentielles: le juge constitutionnel ne souhaite pas infliger le camouflet de l'irrecevabilité à une question dont la transmission a été jugée conforme par les juridictions du filtre. Dès lors, il semble de loin préférer cette technique à celle de l'irrecevabilité et ce même lorsqu'il apprécie non pas la recevabilité d'un moyen à l'occasion de l'examen au fond de la question, mais la recevabilité de la question dans son ensemble au regard des critères posés par la loi organique. En se livrant à l'examen 
de la "validité de la saisine du Conseil constitutionnel ${ }^{31}$ ce dernier juge volontiers, sous l'intitulé "Sur la recevabilité », que la question est recevable ${ }^{32}$, il conclut cependant rarement qu'elle est irrecevable, quand bien même tout l'indiquerait ${ }^{33}$. Il ne s'y résout que dans une hypothèse, celle où la question n'a pas été posée à l'occasion d'une instance en cours $^{34}$.

Le Conseil constitutionnel mobilise avec moins de réticence le vocable de l'inopérance, celui-ci apparaissant dans 77 décisions sur l'ensemble du corpus étudié ${ }^{35}$, soit un peu plus de $10 \%$ des décisions. Cette régularité n'éteint pas pour autant les problématiques posées par cette catégorisation: ce sera là notre quatrième point. La difficulté ne provient pas tant des cas où la motivation identifie expressément l'inopérance que des motivations qui, tout en adoptant une approche très similaire, ne partagent pas cette terminologie. Ainsi par exemple des cas dans lesquels le Conseil constitutionnel considère que les dispositions contestées ne portent pas atteinte "tant par leur objet que par leur portée " aux normes constitutionnelles invoquées ${ }^{36}$. Ici, la consécration d'un moyen inopérant apparaissait logique tant la formulation apparaît proche de la signification usuelle de cette notion. C'est encore le cas lorsque les juges de la rue Montpensier qualifient l'invocabilité d'un objectif de valeur constitutionnelle d'irrecevable lorsqu'il n'est pas invoqué au soutient d'une atteinte à un droit et liberté que la Constitution garantit ${ }^{37}$. L'usage pourtant rare de l'irrecevabilité surprend ici. En effet, on comprend que la dimension téléologique de l'objectif n'implique pas qu'il puisse servir de fondement, "en lui-même", pour démontrer l'inconstitutionnalité de l'atteinte. Toutefois conclure à l'irrecevabilité plutôt qu'à l'inopérance ne semble pas idéal pour plusieurs raisons. Tout d'abord il le fait dans de très rares cas en privilégiant à propos des objectifs de valeur constitutionnelle la formule suivant laquelle ils ne peuvent, en eux-mêmes, être invoqués au soutien d'une violation, sans pour autant consacrer explicitement l'irrecevabilité du moyen lorsqu'ils sont invoqués isolément ${ }^{38}$. En outre, l'objectif de valeur constitutionnelle peut, d'une part, être invoqué au soutien de l'atteinte à une liberté ${ }^{39}$, tandis qu'il n'est pas exclu d'autre part qu'un objectif, à l'égard d'une disposition législative spécifique, puisse susciter le relevé d'office d'un moyen par le juge constitutionnel. En définitive, il semble que l'invocation d'un objectif de valeur constitutionnelle par le requérant appelle nécessairement une appréciation par le juge de la capacité du moyen à mettre en évidence une éventuelle inconstitutionnalité au regard des droits et libertés que la Constitution garantit. Ce contrôle de l'adéquation paraît relever plus naturellement de l'inopérance que de l'irrecevabilité, pourtant ponctuellement employée par le Conseil constitutionnel.

Ces difficultés sont réelles et compliquent à n'en pas douter la tâche de systématisation visant à proposer une typologie des moyens non examinés au fond par le Conseil constitutionnel dans le cadre des décisions QPC. Pourtant il ne faut pas céder à l'exagération, les difficultés évoquées ne rendent pas tout effort de catégorisation impossible.

\section{B. Quelques pistes en vue d'une distinction opératoire}

Le contentieux de la question prioritaire de constitutionnalité revêt certainement quelque originalité qui interdit de supposer un mimétisme parfait avec la jurisprudence administrative. Nous avons vu que cette singularité n'allait pas sans poser un problème à l'observateur soucieux de systématiser l'œuvre jurisprudentielle, en cherchant par exemple à proposer une typologie des moyens non examinés au fond. Il ne nous semble 
cependant ni réaliste ni pragmatique de dramatiser la situation. Ce n'est pas réaliste car dans la grande majorité des cas, les moyens non examinés au fond apparaissent de manière explicite dans la jurisprudence, et le fait que le Conseil les affuble de qualificatifs divers n'estompe pas, en définitive, leur rattachement aux catégories qui nous occupent. Cela ne semble pas non plus pragmatique puisqu'il apparait à bien des égards utile de disposer de catégories descriptives globalement opératoires, quand bien même elles apparaitraient inaptes à recouvrir quelques cas particuliers. L'examen de l'exception, aussi judicieux et informatif soit-il, ne doit pas masquer l'existence de la régularité avec laquelle il rompt. Bien au contraire, il apparaît que la première ne tire son intérêt que de la seconde ${ }^{40}$.

Or, s'agissant des moyens non examinés au fond dans le cadre du contentieux de la QPC, il est indubitable que le Conseil a cherché, depuis plus de dix ans, à mettre en œuvre un schéma méthodique ${ }^{41}$. Cette tendance est particulièrement marquée dans deux domaines : celui relatif à la validité de la saisine du Conseil constitutionnel ${ }^{42}$ et celui de la capacité d'un moyen à démontrer l'atteinte réalisée par la disposition contestée et la norme constitutionnelle invoquée. Ces deux questions nous paraissent respectivement relever d'une distinction opératoire entre irrecevabilité et inopérance dans le cadre du contentieux de la QPC. Revenons brièvement à la théorie des moyens irrecevables et à la théorie des moyens inopérants pour en rendre compte.

Stéphanie Douteaud, dans un article consacré à cette distinction en droit administratif, dresse un constat relativement sévère de son état, jurisprudentiel comme doctrinal ${ }^{43}$. Elle constate que "l'indétermination de la frontière entre moyen inopérant et moyen irrecevable découle de l'impuissance des acteurs du contentieux administratif à dégager les éléments constitutifs de chacun de ces « états » du moyen » et en impute la faute au juge, dès lors que ce dernier a la responsabilité sinon exclusive, du moins principale, de définir juridiquement les termes de son contrôle ${ }^{44}$. La doctrine n'est pas parvenue à suppléer à cette carence de la jurisprudence en proposant d'indexer la distinction soit sur le moment où le rejet intervient ${ }^{45}$ soit sur l'influence qu'exerce l'auteur de l'action sur le sort du moyen ${ }^{46}$. La première hypothèse est insatisfaisante en ce que la consécration d'un moyen irrecevable comme celle d'un moyen inopérant intervient toujours en amont de l'examen au fond du moyen ${ }^{47}$. Nous croyons devoir attester de la même convergence dans le contentieux constitutionnel, raison pour laquelle nous englobons ces qualificatifs sous le label plus général de moyens "non examinés au fond». La seconde hypothèse souffre quant à elle d'une incompatibilité avec la pratique du contrôle de l'irrecevabilité. Il apparaît en effet que l'auteur de l'action n'a guère d'influence sur la compétence du juge administratif alors même que son incompétence est un motif patent d'irrecevabilité des moyens. Stéphanie Douteaud envisage donc une théorie alternative à même de surmonter ces difficultés.

Elle propose de fonder la distinction en considérant que l'«inopérance découle d'un contrôle orienté vers les effets que le moyen serait à même de produire dans l'hypothèse où il serait examiné » tandis que "l'irrecevabilité est établie en fonction de l'examen d'éléments antérieurs à l'introduction de l'instance $»^{48}$. En ce sens, le contrôle de l'opérance d'un moyen imposerait d'examiner « la capacité du moyen à satisfaire la demande de l'auteur du recours $»^{49}$. Il impose donc un contrôle prospectif au juge qui doit appréhender les conséquences potentielles de son contrôle. Il conclut dès lors à l'inopérance lorsque "accueillir le moyen serait sans conséquence sur le sort du requérant $\aleph^{50}$. A l'inverse, le contrôle de l'irrecevabilité impliquerait une enquête 
rétrospective puisque sa reconnaissance serait fondée sur l'identification d'«un élément qui préexiste à l'invocation du moyen $»^{51}$ et qui ferait obstacle à son examen au fond.

Cette proposition présente à notre sens plusieurs vertus. Premièrement, elle rend compte de l'intuition jurisprudentielle et doctrinale suivant laquelle inopérance et irrecevabilité sont des motifs de rejets strictement distincts. Un moyen peut être inopérant et recevable ou encore opérant et irrecevable. Accessoirement elle ne contredit pas l'idée générale qui veut qu'inopérance et irrecevabilité entrainent alternativement ou cumulativement - le non-examen au fond du moyen et qu'a contrario, l'examen du bien-fondé du moyen suppose qu'il soit cumulativement opérant et recevable. Deuxièmement, la distinction présente l'avantage de se conformer à la grande majorité des usages jurisprudentiels comme aux catégorisations doctrinales canoniques. Troisièmement, elle présente un intérêt indubitable pour l'étude du contentieux constitutionnel qui nous intéresse.

21 En effet, les décisions QPC illustrent bien cette distinction entre un contrôle rétrospectif appréciant la nécessité d'examiner le moyen au regard d'éléments préexistants à l'instance et un contrôle prospectif fondé sur la capacité du moyen à satisfaire la finalité du recours. Le contrôle rétrospectif se présente, devant le juge de la QPC, sous les traits du contrôle de la validité de sa saisine. Il se comprend ainsi au travers du respect des règles de procédures et de compétences applicables à la question prioritaire de constitutionnalité. On assimilera ce contrôle de la validité de la saisine au contrôle de la recevabilité du moyen; et ce, quand bien même la haute instance le présenterait à l'aide d'une terminologie distincte, comme celle du non-lieu à statuer ${ }^{52}$. Parallèlement, le juge constitutionnel se livre dans le cadre de la question prioritaire de constitutionnalité à un examen de l'aptitude du moyen à engendrer l'inconstitutionnalité recherchée. Ce contrôle de l'opérance est préalable à l'examen au fond et rend, par conséquent, ce dernier caduc lorsque l'inopérance est constatée. Là encore, cette seconde catégorisation peut certes, à la marge, impliquer de défier la terminologie expressément adoptée par le Conseil, mais il nous semble que, dans la très large majorité des cas, l'identification d'un moyen inopérant s'accompagne de l'usage du terme idoine.

\section{II- Les différentes formes de moyens irrecevables et inopérants en QPC}

Fort de cette distinction il apparaît maintenant possible d'étudier les formes topiques que revêtent le contrôle de la recevabilité (A) et le contrôle de l'opérance (B) dans le contentieux de la question prioritaire de constitutionnalité.

\section{A. Le contrôle de l'irrecevabilité : des moyens inaptes à saisir valablement le Conseil constitutionnel}

23 Le Conseil constitutionnel s'appuie sur un contrôle rétrospectif pour apprécier la validité de sa saisine, et potentiellement conclure à son irrecevabilité totale ou partielle, dans quatre cas de figures: en raison de l'autorité des décisions du Conseil 
constitutionnel (1), en raison de son incompétence (2), lorsque la question apparaît viciée formellement (3) ou procéduralement (4).

\section{1) L'irrecevabilité fondée sur l'autorité des décisions du Conseil constitutionnel} D'une part, il entend garantir l'autorité de ses décisions en s'appuyant sur le principe non bis in idem tandis que, d'autre part, il ne souhaite pas conférer de brevet de constitutionnalité irréfragable à des dispositions qu'il n'aurait pas dument examinées ou qui seraient appelées à évoluer en raison d'un changement de circonstances de droit ou de fait. Pour justifier du réexamen d'une disposition, le juge est contraint, par la nature objective de son contrôle, de s'appuyer le plus souvent sur un changement de circonstances de droit ${ }^{55}$ quoi qu'il se soit, en une occasion au moins, basé sur des circonstances de fait ${ }^{56}$. Par ailleurs la haute instance retient que le principe non bis in idem ne s'applique que pour les dispositions ou les extraits de dispositions expressément déclarés conformes dans les motifs et dans le dispositif ${ }^{57}$. Enfin, le Conseil constitutionnel tend à favoriser la reconnaissance d'un changement de circonstances en reconnaissant qu'un tel changement peut découler d'une évolution de la jurisprudence du Conseil constitutionnel ${ }^{58}$ ou des cours suprêmes ${ }^{59}$.

La consécration d'un changement de circonstances peut cependant se trouver empêchée par divers éléments entraînant l'irrecevabilité totale ou partielle de la question sur le fondement du principe non bis in idem ${ }^{60}$. Lorsque ce principe s'impose, le Conseil jugera dès lors qu'il n'y a pas lieu de statuer sur la question, et ce, que la situation juridique acquise soit survenue entre l'enregistrement de la question et la décision ${ }^{61}$, ou antérieurement à l'enregistrement de la question ${ }^{62}$. A ce titre, si la jurisprudence constante des cours suprêmes est susceptible d'emporter un changement de circonstances, il n'en va pas de même de la jurisprudence des juges du fond qui ne serait pas confirmée en ces termes ${ }^{63}$. De même, le Conseil constitutionnel peut se livrer à un raisonnement analogique pour considérer qu'il n'y a pas lieu de statuer lorsqu'est contestée une disposition «[non examinée] par le Conseil constitutionnel mais dont la rédaction est strictement identique à une disposition expressément validée ${ }^{64}$. Enfin, il peut librement considérer, au rebours de la position de la juridiction de renvoi, qu'une évolution factuelle n'est pas constitutive d'un changement de circonstances justifiant le réexamen d'une disposition ${ }^{65}$.

définitive, si la théorie des changements de circonstances est indubitablement un facteur de « renouvellement » et d'« enrichissement » du contrôle de constitutionnalité a posteriori ${ }^{66}$, sa portée demeure entre les mains des juridictions du filtre et du Conseil constitutionnel, qui l'utilise aussi comme un moyen de filtrer les questions qui lui sont transmises et d'asseoir l'autorité de ses décisions.

Un autre genre d'irrecevabilité, plus marginal, nous paraît également s'inscrire dans cette même logique. Il s'agit des demandes de rectification d'erreur matérielle qui sont 
rejetées au motif qu'elles se présentent en vérité comme des remises en cause substantielles des décisions de QPC ${ }^{67}$.

Lorsque ce n'est pas l'expression antérieure de la compétence du Conseil qui fonde sous l'égide du principe non bis in idem, l'irrecevabilité, ce peut être, au contraire, l'expression de son incompétence.

\section{2) L'irrecevabilité fondée sur l'incompétence du Conseil constitutionnel}

30 Le Conseil constitutionnel juge aussi irrecevable un moyen en ce qu'il conteste une disposition législative à l'égard de laquelle il est incompétent pour en connaître. Ce pan de l'irrecevabilité des décisions QPC manifeste un parallélisme certain avec la jurisprudence établie dans le cadre du contrôle a priori.

31 Ainsi les juges de la rue Montpensier se refusent à connaître de la constitutionnalité d'une disposition référendaire ${ }^{68}$, appliquant strictement la jurisprudence constante née en 1962 suivant laquelle le fait que ces lois "constituent l'expression directe de la souveraineté nationale " les soustrait à son appréciation ${ }^{69}$. Il en va également ainsi des lois qui "se bornent à tirer les conséquences nécessaires de dispositions inconditionnelles et précises d'une directive de l'Union européenne " sans "mise en cause d'une règle ou d'un principe inhérent à l'identité constitutionnelle de la France $»^{70}$. Ce motif de rejet reprend littéralement les termes conduisant à la même solution dans le cadre du contrôle a priori $^{71}$ et emporte les deux mêmes exceptions. L'analyse a contrario du considérant révèle en effet que le Conseil est compétent, et partant la question recevable, si la disposition contestée n'est pas une transposition directe de la directive ${ }^{72}$, ou si la loi porte atteinte à un principe inhérent à l'identité constitutionnelle de la France ${ }^{73}$. On remarquera ici que cette dernière motivation pose un problème en termes de classification. En effet, traditionnellement la "[critique d'] une mesure que son auteur est dans l'obligation d'adopter ${ }^{74}$ relève du contrôle de l'inopérance et non de l'irrecevabilité. Il semble néanmoins que dans ce cas spécifique l'une rejoigne l'autre, l'incompétence juridictionnelle découlant du caractère obligatoire de la transposition contestée.

32 Ces motifs d'irrecevabilité sont à rapprocher des cas de figure dans lesquels le Conseil constitutionnel rejette le moyen au motif que la question ne vise pas la contestation d'une disposition législative. Toutefois, ce n'est plus dès lors l'incompétence du Conseil qui est mise en avant mais le vice formel de la question.

\section{3) L'irrecevabilité fondée sur le moyen formellement vicié}

33 Le Conseil constitutionnel s'autorise à désavouer la juridiction de renvoi en estimant qu'un moyen est irrecevable lorsqu'il constate que la question ne porte pas sur une disposition législative.

34 C'est classiquement le cas lorsque la disposition visée est de nature règlementaire ${ }^{75}$. On notera toutefois que ce motif de rejet connait un tempérament en ce que le Conseil constitutionnel s'autorise à contrôler les dispositions règlementaires annexées à des lois $^{76}$. Suivant la logique de partition entre le contrôle de la loi et celui du règlement, les ordonnances non ratifiées se voient logiquement exclues du contentieux de la QPC, en tant qu'elles possèdent une valeur règlementaire ${ }^{77}$. Depuis la décision du 28 mai $2020^{78}$ le Conseil retient toutefois que les ordonnances non ratifiées acquièrent automatiquement la valeur de dispositions législatives une fois écoulée la période d'habilitation. Cette décision présente l'avantage de favoriser la contestation de ces 
ordonnances par le truchement de la question prioritaire de constitutionnalité mais soulève d'évidentes interrogations quant à la souveraineté du Parlement et à sa conformité à l'art. $38 \mathrm{C}$ al. 2 qui énonce explicitement que les ordonnances «ne peuvent être ratifiées que de manière expresse ${ }^{79}$.

Outre le cas des dispositions n'ayant pas une valeur législative, le Conseil constitutionnel déclare également le moyen irrecevable en tant qu'il apparaît formellement vicié : s'il conteste non pas une disposition législative précise mais une loi dans son ensemble, en invoquant au soutien de l'inconstitutionnalité une large part du bloc de constitutionalité ${ }^{80}$. Le commentaire officiel sous cette décision précise utilement que «l'applicabilité au litige dont l'auteur de la QPC doit se prévaloir pour que celleci soit recevable est un lien effectif et concret entre la procédure à laquelle il est partie et une disposition législative. Il ne suffit pas davantage, pour poser valablement une QPC, de faire "feu de tout bois " constitutionnel en invoquant l'ensemble du bloc de constitutionnalité et même audelà $»^{81}$. Dans cette décision, à notre connaissance unique, le Conseil constitutionnel se fait ostensiblement le juge des conditions de recevabilité de sa saisine, quand bien même le commentaire officiel le nierait farouchement ${ }^{82}$ en reconnaissant avec les juges qu'il n'y a simplement pas lieu à statuer.

"trop plein » de dispositions législatives suscite l'irrecevabilité, l'absence de disposition législative amène un résultat identique. Ainsi, le fait de ne pas mentionner l'une des dispositions législatives entraînant un cumul des poursuites constitutif de l'inconstitutionnalité engendre l'irrecevabilité de la question ${ }^{83}$, il en va de même si la disposition contestée n'est jamais entrée en vigueur ${ }^{84}$, ou de toute évidence, si le moyen est dirigé contre une décision de justice et non une disposition législative ${ }^{85}$.

Le moyen apparaît tout aussi irrecevable sur la forme lorsqu'il invoque une atteinte à autre chose qu'un droit ou une liberté garantie par la Constitution ${ }^{86}$. Cette catégorie est en fait particulièrement ambigüe et comme on le verra, elle nous parait englober des moyens irrecevables et des moyens inopérants. Des moyens irrecevables lorsque la norme invoquée n'est pas un droit ou une liberté que la Constitution garantit, des moyens inopérants lorsque la norme consacre un droit ou une liberté mais que celle-ci est inerte quant au grief soulevé par le requérant. Le problème tient bien sûr au fait que la motivation adoptée par le Conseil constitutionnel entretient une confusion importante relativement à cette partition.

38 En effet, celui-ci écarte par exemple explicitement les normes relatives à la procédure d'adoption de la loi des normes invocables dans le champ de la $\mathrm{QPC}^{87}$ la justification d'une telle limitation serait que «le Conseil constitutionnel exclut ainsi du champ de la QPC les dispositions qui ne s'adressent qu'au législateur ou aux autorités publiques et dont les justiciables ne sont que les destinataires indirects ${ }^{88}$. Cette formulation laisse entendre que les moyens fondés sur ces dispositions constitutionnelles sont irrecevables en vertu de l'objet même de ces normes, qui n'est pas «directement » le justiciable. Pourtant, le trouble est jeté par l'évocation, quelques lignes plus haut, de la notion de droits et libertés que la Constitution garantit : «le Conseil constitutionnel a en conséquence jugé que cette formulation, destinée à circonscrire le périmètre des moyens de constitutionnalité invocables, exclut du contrôle a posteriori différentes normes constitutionnelles bien qu'elles soient opérantes dans le cadre du contrôle a priori»" ${ }^{89}$. Partant, l'ambivalence de la formulation ne permet pas de déterminer si de tels moyens sont écartés en raison de leur irrecevabilité ou de leur inopérance. Si la confusion apparaît totale dans la motivation de la décision, elle l'est également pour son observateur. 
39 Le refus de rattacher une norme constitutionnelle à la catégorie des droits et libertés que la Constitution garantit relève-t-il de l'irrecevabilité ou de l'inopérance? Nous ferions cette proposition. Si la norme est susceptible d'être invoquée au soutien de la violation d'un droit ou d'une liberté que la Constitution garantit, mais qu'un tel rattachement n'est pas constaté en l'espèce, alors son rejet relève de l'inopérance ${ }^{90}$. A l'inverse, si la norme n'est jamais susceptible d'être invoquée, alors le moyen qui s'en prévaut est irrecevable. Cette dernière hypothèse s'illustre dans le cas des normes relatives à la procédure d'adoption de la loi, mais aussi de certains principes consacrés par le bloc de constitutionnalité, comme le droit de consentir à l'impôt ${ }^{91}$ ou le principe suivant lesquelles "les collectivités territoriales ont vocation à prendre les décisions pour l'ensemble des compétences qui peuvent le mieux être mises en oeuvre à leur échelon ${ }^{92}$. Elle se présente également lorsque le Conseil constitutionnel écarte la consécration d'un principe nouveau comme le "principe de publicité des séances et des votes lors des délibérations des assemblées locales ${ }^{93}$ ou d'un PFRLR, selon lequel "l'exclusivité des droits patrimoniaux attachés à une cuvre intellectuelle doit nécessairement s'éteindre après l'écoulement d'un certain délai $»^{94}$ ou encore "imposant au législateur de prévoir un délai de prescription de l'action publique pour les infractions "dont la nature n'est pas d'être imprescriptible $" \|^{95}$.

40 Lorsque l'irrecevabilité ne découle pas d'un vice imputable à la disposition contestée ou à la norme invoquée, c'est alors un défaut de procédure qui peut en être à l'origine.

\section{4) L'irrecevabilité fondée sur le vice de procédure}

41 Ce dernier motif se distingue des autres en ce que grevant la question dans son ensemble il engendre nécessairement l'irrecevabilité totale de la question là où les figures précédemment évoquées pouvaient, au gré des moyens concernés par l'irrecevabilité, déboucher sur une irrecevabilité partielle.

42 L'irrecevabilité pour vice de procédure se pose à l'occasion de questions dont le Conseil est directement saisi par les requérants, faute d'un renvoi en bonne et due forme par la juridiction suprême concernée. Il nous semble intéressant de relever qu'en pratique «le Conseil constitutionnel est assez régulièrement destinataire de demandes émanant de personnes qui tentent de se prévaloir des dispositions de l'article 23-7 de l'ordonnance du 7 novembre 1958 pour obtenir que le Conseil constitutionnel examine directement une $Q P C »^{96}$. Toutefois, ces questions apparaissent dans la plupart des cas manifestement irrecevables et le requérant se voit informé de l'extinction de la procédure en QPC sans que le Conseil constitutionnel ne se réunisse solennellement ${ }^{97}$. De rares exemples illustrent cependant la réunion des conditions de l'art. $23-7^{98}$, d'autres, tout aussi rares ${ }^{99}$, donnent lieu à une décision d'irrecevabilité solennelle du Conseil constitutionnel.

43 La décision $n^{\circ}$ 2012-237 QPC présente un cas particulier dans lequel un requérant a soulevé une QPC devant la juridiction ordinaire avant d'en soulever une seconde devant la Cour de cassation. Après transmission de la première QPC, la Cour de cassation se trouvait donc confronté à deux questions prioritaires de constitutionnalité. Or, elle n'a explicitement refusé de renvoyer que la QPC qui avait été soulevée devant elle ${ }^{100}$, en conséquence, le requérant a cru pouvoir saisir le Conseil constitutionnel sur le fondement d'un dessaisissement automatique de la Cour de cassation s'agissant de la première question. Le juge constitutionnel a considéré que cette question était identique puisqu'elle déployait les mêmes griefs, par le même requérant, à l'encontre 
de la même disposition ${ }^{101}$. Il fallait donc considérer que le refus de renvoyer valait pour les deux questions et il conclut à l'irrecevabilité de la saisine directe sur ce fondement.

La décision $n^{\circ}$ 2014-440 QPC pose l'épineux problème des instances éligibles à la QPC ${ }^{102}$. On sait que la loi organique prévoit en son article 23-1 alinéa 1 que la question prioritaire de constitutionnalité doit être posée devant une juridiction relevant de la Cour de cassation ou du Conseil d'État. Or, une interrogation ressortait en l'espèce des questions posées consécutivement aux demandes d'aide juridictionnelle car les décisions du bureau d'aide juridictionnelle peuvent être déférées, entre autres et selon les cas, aux présidents de la Cour d'appel, de la Cour de cassation, ou de la section du contentieux du Conseil d'État ${ }^{103}$, la question de savoir si ce recours constituait une instance en cours au sens de l'art. 61-1 C se posait donc. La Cour de cassation (comme le Conseil d'État) a toujours refusé de transmettre une question posée à l'occasion d'un recours contre une décision $d u$ bureau d'aide juridictionnelle ${ }^{104}$. Le Conseil constitutionnel n'a fait qu'entériner cette solution dans sa décision n 2014-440 QPC en retenant que « la procédure d'admission à l'aide juridictionnelle [...] n'est pas une instance en cours à l'occasion de laquelle une question prioritaire de constitutionnalité peut être posée ». Si l'on comprend que le Conseil ait tenté par cette décision de tarir le flot de QPC manifestement irrecevables dont il est saisi, on regrettera cependant qu'il n'ait pas accueilli la demande en vue d'un examen au fond. En effet, le rejet de la demande d'aide juridictionnelle conduit en pratique à priver certains requérants potentiels de tout recours. Partant les questions que le Conseil constitutionnel invite par cette décision à poser ailleurs n'auront, en définitive, peut être aucune réponse.

Les différentes formes d'irrecevabilité exposées supposent toutes, on l'a vu, de mettre en cause, au moyen d'un examen rétrospectif, la validité de la saisine du Conseil constitutionnel. Soit le moyen contrevenait à l'autorité de la chose jugée par le Conseil, soit il manquait de relever son incompétence, soit il ne contestait pas validement une disposition législative, soit il omettait un vice procédural condamnant la recevabilité de la question. Il convient maintenant de s'interroger sur les moyens rejetés à l'issue d'un contrôle prospectif constatant leur inopérance.

\section{B. Le contrôle de l'inopérance : des moyens inaptes à déterminer l'atteinte invoquée}

Rappelons que le moyen inopérant s'entend comme celui qui n'est pas susceptible de déterminer l'issue du litige. En ce sens, le moyen est jugé inopérant lorsque son invocation par le justiciable est "inoffensive ${ }^{105}$ à l'égard de l'issue du litige. On identifiera deux catégories de motivations mobilisées par le Conseil constitutionnel et relevant de cette logique : les cas dans lesquels la ou les norme(s) constitutionnelle(s) invoquée(s) ne consacre(nt) pas un droit ou une liberté invocable par le justiciable et ceux dans lesquels la disposition contestée ne porte pas atteinte au droit ou à la liberté invoquée.

Dans le premier cas de figure, le caractère inoffensif nait donc de l'incapacité pour le requérant de se prévaloir du «bon » droit (ie. moyen) invoqué, dans le second cas, ce sont les effets de la disposition législative invoquée qui sont inertes à l'encontre du droit (ie. moyen), dont le requérant peut pourtant légitimement se prévaloir. 


\section{1) L'inopérance découlant de la non-invocabilité de certaines normes constitutionnelles}

48 La première catégorie, on l'a vu plus haut, tend à se confondre avec un motif d'irrecevabilité du moyen en ce qu'elle appartient à la catégorie plus générale des normes qui ne consacrent pas un droit ou une liberté que la Constitution garantit au sens de l'art. 61-1 C. Pourtant, une distinction nous semble devoir prospérer au sein de cette dénomination. En effet, s'agissant de l'incompétence négative, des objectifs de valeur constitutionnelle, ou de certains principes consacrés par le bloc de constitutionnalité106, le rejet du moyen les invoquant n'apparaît pas catégorique et absolu contrairement, par exemple, aux normes encadrant la procédure législative. Cela se matérialise notamment par le fait que le Conseil constitutionnel indique expressément que le moyen est précocement rejeté soit au regard du grief invoqué, soit parce qu'il n'est pas "en lui-même", susceptible d'être invoqué dans le cadre de la question prioritaire de constitutionnalité. Cette nuance nous paraît déterminante pour distinguer les moyens irrecevables des moyens inopérants. En effet, la référence explicite au grief invoqué par le requérant comme la possibilité d'une conjonction susceptible de rendre le moyen opérant nous parait caractéristique de l'inopérance. Pour le dire simplement, lorsque le Conseil constitutionnel juge qu'une norme n'est pas invocable en elle-même au soutien d'une QPC ou ne consacre pas un droit ou une liberté garantie par la Constitution au regard des griefs invoqués, il semble déceler une inaptitude contingente du moyen à influencer l'issue du litige, et non une inaptitude absolue et pérenne valant pour l'ensemble des QPC. En ce sens, ces tournures apparaissent caractériser des moyens inopérants ${ }^{107}$.

On les retrouve typiquement dans les questions invoquant l'incompétence négative du législateur en négligeant de démontrer le lien entre la violation de l'art. $34 \mathrm{C}$ et un droit ou une liberté garantie par la Constitution, soit que le droit et liberté auquel il est porté atteinte ne soit pas invoqué ${ }^{108}$, soit que l'atteinte à ce droit ne soit pas caractérisée ${ }^{109}$. Le juge constitutionnel apprécie cette conjonction de manière particulièrement fine puisqu'il peut considérer, au sein d'une même décision, que la méconnaissance par le législateur de l'étendue de sa compétence dans un domaine donné n'affecte pas certains droits et libertés tandis qu'elle en affecte d'autres ${ }^{110}$. Une stratégie argumentative voisine est appliquée aux objectifs de valeur constitutionnelle dont l'invocation est jugée inopérante lorsqu'elle n'est pas associée à l'atteinte portée à un droit ou une liberté. Il en va ainsi de l'objectif d'intelligibilité et d'accessibilité de la $\operatorname{loi}^{111}$, de l'objectif de sauvegarde de l'ordre public ${ }^{112}$, de l'objectif de lutte contre la fraude et l'évasion fiscale ${ }^{113}$, de l'objectif de bon usage des deniers publics ${ }^{114}$ ou de l'objectif de bonne administration de la justice ${ }^{115}$.

Par cette limite le Conseil constitutionnel nous semble avoir cherché à contenir l'invocabilité systématique de l'incompétence négative ou des objectifs de valeur constitutionnelle. Le caractère relativement vague de leur formulation et leur dimension par définition prospective favorisent en effet une invocabilité très large de ces ressources constitutionnelles. L'encadrement proposé vise à ne pas réduire absolument le potentiel qu'elles représentent pour les justiciables comme pour le juge de la constitutionnalité tout en permettant un rejet automatique des mobilisations considérées comme fantaisistes et manifestement injustifiées ${ }^{116}$. 


\section{L'inopérance découlant de l'absence d'atteinte aux droits et libertés invoqués}

51 La seconde catégorie est quantitativement la plus fréquente et représente le cas typique de l'inopérance relevée en l'absence de rapport (ou de lien visible) entre la disposition contestée et la norme constitutionnelle invoquée. Dans cette hypothèse, l'inopérance découle donc de l'incapacité de la disposition contestée à porter atteinte au droit ou à la liberté invoquée. Cette rhétorique connait une illustration sérielle s'agissant de l'invocation des droits de la défense et des principes constitutionnels régissant le droit pénal, le Conseil constitutionnel retenant qu'un tel grief est inopérant s'agissant des "sanctions n'ayant pas le caractère d'une punition "117. Cette technique se retrouve dans le domaine électoral, lorsque le juge identifie un moyen inopérant en considérant que la présentation des candidatures à l'élection présidentielle ne constitue pas l'expression d'un suffrage et ne peut donc affecter le secret du suffrage ${ }^{118}$. Dans la même, logique, le juge n'hésite pas à déclarer inopérant un moyen tiré du droit d'obtenir un travail consacré par le cinquième alinéa du préambule de 1946 au motif que les mandats électifs ne sont pas des emplois ${ }^{119}$. Il rappelle par ailleurs que l'aide financière aux partis politiques, en ce qu'elle ne profite aux parlementaires que de manière contingente, ne peut porter atteinte au principe d'égalité entre les membres du Parlement ${ }^{120}$. En outre, le juge est régulièrement conduit à rejeter comme inopérant des moyens tirés de la Charte de l'environnement en ce qu'elles seraient sans lien avec les dispositions législatives contestées ${ }^{121}$.

Le juge peut aussi adopter une justification analogue pour prononcer le rejet sans indiquer clairement que le moyen est inopérant. Le parallélisme des formes invite alors à dresser un constat d'inopérance même si le juge s'en abstient explicitement. En ce sens, la liberté individuelle est régulièrement le théâtre de ce conflit entre les prétentions du requérants et l'autorité interprétative du Conseil constitutionnel. Ce dernier n'hésitant pas à rejeter le grief qui se fonde sur la liberté individuelle au motif que la disposition contestée, et le régime qu'elle emporte, n'y porte pas atteinte. Toutefois, son raisonnement n'est pas toujours accompagné d'un constat d'inopérance expresse $^{122}$, quoiqu'il en présente toutes les conditions, notamment lorsqu'il juge, d'une manière à notre sens contestable, que l'audition sous contrainte des témoins ne méconnait pas les droits de la défense ${ }^{123}$. Cette inopérance implicite se présente également en droit des collectivités territoriales lorsqu'il considère que le processus de fusion des communes n'affecte pas la souveraineté nationale ${ }^{124}$.

Un dernier cas de figure interpelle, il réside dans les décisions retenant que la méconnaissance des exigences constitutionnelles dans l'application des dispositions législatives n'est pas de nature à entacher la disposition d'inconstitutionnalité ${ }^{125}$. Une telle interprétation revient à déclarer inopérant tout moyen fondé sur l'application concrète des dispositions législatives contestées. Elle se justifie bien évidemment par la conception abstraite que le juge constitutionnel se fait de son office. Cependant, elle ne manque pas de priver quelque peu la question prioritaire de constitutionnalité d'un effet utile auquel le juge parait par ailleurs attaché. La contradiction ne semble pouvoir être levée qu'au prix d'un renoncement à la dimension abstraite du contrôle, mais un tel renoncement n'est-il pas ancré dans la vocation originelle de la procédure? Il convient d'en discuter plus avant en interrogeant, en guise de conclusion les incidences du contrôle de l'irrecevabilité et de l'inopérance sur les différents acteurs de la QPC. 


\section{Conclusion}

d'un moyen. On a pu relever dans le corpus étudié 36 décisions de non-lieu à statuer, totaux ou partiels, et 77 décisions relevant un ou plusieurs moyens inopérants. Même si l'on ajoute à ce calcul les décisions dans lesquelles le Conseil constitutionnel semble consacrer implicitement un moyen irrecevable ou inopérant ${ }^{126}$, leur somme demeure relativement faible. Nous en déduisons donc que le Conseil constitutionnel fait peu usage de son pouvoir de troisième filtre et répond, dans la très grande majorité des cas, à la question qui lui est posée. Toutefois, l'étude du contrôle de l'irrecevabilité et de l'inopérance laisse apparaître que si la réponse n'est pas toujours celle attendue par le justiciable, ce qui ne doit pas surprendre, elle n'est pas toujours non plus celle que la procédure de la question prioritaire de constitutionnalité à vocation à appeler, ce qui étonne davantage. Ainsi, indépendamment du prononcé de la censure ou de la conformité des dispositions contestées, la procédure de la question prioritaire de constitutionnalité requiert un examen de la constitutionnalité de ces dispositions caractérisées par une certaine envergure rhétorique et doté d'un minimum d'intensité justificative dans la motivation. Or, les techniques de l'irrecevabilité et de l'inopérance conduisent en pratique à contourner ces impératifs. Elles devraient, de ce seul fait, être marquée par une motivation explicite et cohérente à même d'assurer leur lisibilité par les différents acteurs de la question prioritaire de constitutionnalité. Force est de constater que ce n'est pas rigoureusement le cas.

56 Une telle conclusion appelle quelques précisions. Tout d'abord, il ne faut pas négliger l'incidence des décisions employant ces techniques, aussi rares soient-elles. Elles sont en effet lourdes de sens pour les juridictions du filtre ${ }^{127}$ qu'elles désavouent ouvertement. Identifier un moyen irrecevable ou inopérant pour le Conseil constitutionnel c'est toujours indirectement considérer que la juridiction de renvoi n'a pas correctement appliqué les critères de la loi organique. Quand bien même la haute instance le ferait avec «diplomatie $»^{128}$, «politesse $»^{129}$ ou «pudeur $»^{130}$, il n'en demeure pas moins que de telles décisions contredisent l'appréciation consentie par les juridictions du filtre.

57 En outre, leur rareté s'explique par le fait qu'elles opèrent comme filtrage de dernier ressort, alors même que seules $25 \%$ des questions sont renvoyées par le Conseil d'État devant le Conseil constitutionnel, et $11 \%$ par la Cour de cassation ${ }^{131}$. En vérité, l'essentiel des constats d'irrecevabilité et d'inopérance sont prononcés devant les juridictions ordinaires et les juridictions suprêmes. Toutefois, la jurisprudence formulée par le Conseil constitutionnel à l'égard de ce contrôle apparaît structurante : elle s'impose aux juridictions du filtre, sous peine de voir leurs renvois désavoués, et aux requérants, au risque de laisser leur question sans réponse.

L'effet structurant conféré par la jurisprudence du Conseil constitutionnel connait cependant des limites. Il n'a en effet qu'un impact négatif. Les juges de la rue 
Montpensier peuvent juger irrecevable un renvoi trop généreusement admis par les cours suprêmes, ils ne peuvent cependant pas admettre une question que ces dernières n'auraient pas renvoyée. On touche ici aux limites de la procédure de la question prioritaire de constitutionnalité, et plus généralement à l'incapacité pour le Conseil constitutionnel de s'auto-saisir ou d'être saisi directement par les justiciables contestant la constitutionnalité de la loi ${ }^{132}$. La forme même de la procédure suppose que le Conseil constitutionnel est dépossédé d'un pouvoir d'homogénéisation du contentieux qui lui appartient pourtant en théorie, en raison de sa fonction d'interprète authentique de la Constitution. Une réforme en ce sens aurait indubitablement le double mérite de raffermir le contrôle de l'institution sur l'autorité de ses décisions tout en évitant d'exposer l'opportunité du renvoi aux divergences d'interprétation des juridictions ordinaires ${ }^{133}$.

Nous insisterons ensuite sur la forme qu'arbore dans la motivation du Conseil constitutionnel, les arguments d'irrecevabilité et d'inopérance. Cette forme, nous l'avons déploré, est changeante et indéterminée. Elle ne s'inspire que partiellement des catégories de la jurisprudence administrative qu'elle reprend, elle s'applique de manière disparate à des moyens pourtant similaires, elle évolue au gré de la jurisprudence du Conseil, et notamment, mais pas seulement, au gré de l'invocation de changements de circonstances. De plus, cette indétermination est aggravée par le fait que la justification qui amène à déclarer un moyen irrecevable ou inopérant est pour le moins minimaliste, et dans les cas les plus problématiques, le commentaire ne supplée pas à cette brièveté et se borne le plus souvent à rappeler, pour toute explication, la jurisprudence du Conseil constitutionnel ${ }^{134}$.

Ce constat n'a évidemment rien d'inédit et se trouve même aggravé par le principe de l'économie des moyens ${ }^{135}$ que le Conseil fait sienne et par l'attachement à la brièveté de sa motivation ${ }^{136}$. Si celle-ci évolue indubitablement depuis quelques années en faveur d'une plus grande intelligibilité ${ }^{137}$, il ne fait pas de doute que ces progrès n'estompent pas le caractère lapidaire de certaines formulations, tout particulièrement s'agissant des motivations de rejet. On reconnaîtra volontiers, avec P-Y. Gahdoun ${ }^{138}$ que cette imperfection suppose des bienfaits, tout particulièrement du point de vue du Conseil constitutionnel qui y trouve là matière à varier son appréciation au gré des différentes questions qui lui sont soumises. L'ambiguïté est gage, pour lui, de liberté.

61 Cette soumission de l'institution à une conception héraclitéenne de son office, ouverte aux contingences et flexible quant au devenir de sa jurisprudence, ne peut que favoriser sa marge d'appréciation et, imagine-t-on, l'exercice de son autorité. Toutefois, le flou entourant ce contrôle de l'irrecevabilité et de l'inopérance ne peut que déteindre sur la jurisprudence du filtre, qui ne peut prétendre à aucune homogénéité en l'absence d'une objectivation des critères du contrôle. De même, les requérants et leurs conseils ne peuvent qu'être découragés par une procédure laborieuse susceptible de leur échapper à tout instant pour des causes qui ne sont ni toujours de leur ressort ni explicitement motivées. On ne peut alors qu'espérer une nouvelle évolution de la jurisprudence du Conseil constitutionnel qui parviendrait, dans l'intérêt de ces différents acteurs, à définir plus clairement les critères de l'irrecevabilité et de l'inopérance dans le cadre de la question prioritaire de constitutionnalité. Telle serait la condition pour réconcilier, sur ce sujet du moins, Héraclite et Parménide. 


\section{NOTES}

1. La citation exacte est la suivante: «quelles sont les seules voies de recherche qu'il y ait à penser : / la première, que cela est, et qu'il n'est pas possible que cela ne soit pas -/ c'est le chemin de la persuasion, car il suit la vérité ; / la deuxième, que cela n'est pas, et qu'il est nécessaire que cela ne soit pas " (Parménide, fragment B 2, 1-5) cité par Balaudé J-F., Le vocabulaire des présocratiques, éd. Ellipses, 2011, p. 35.

2. Héraclite, fragment 91 ; cité par Brun J., Les présocratiques, éd. PUF, coll. Que sais-je ?, 2019, p. 49.

3. Douteaud S., "Pour une clarification de la distinction entre moyens inopérants et moyens irrecevables en contentieux administratif », in RFDA, 2016, pp. 697-706.

4. V. notamment Girard D., "Le «non lieu à statuer» devant le conseil constitutionnel : un élément révélateur de son ambivalence entre organe politique et cour constitutionnelle ", in Revue française de droit constitutionnel, 2015/1, n 101, pp. e55 à e76, spéc. e56-58.

5. Chapus R., Droit du contentieux administratif, Montchrestien, Domat droit public, 13e éd., 2008, p. 810 ; cité par Douteaud S., «Pour une clarification de la distinction entre moyens inopérants et moyens irrecevables en contentieux administratif », loc. cit., p. 697.

6. A. Ciaudo, L'irrecevabilité en contentieux administratif français, éd. L'Harmattan, Logiques juridiques, 2009, p. 240 ; citée par Douteaud S., «Pour une clarification de la distinction entre moyens inopérants et moyens irrecevables en contentieux administratif », loc. cit., p. 697.

7. Ibid.

8. Ibid.

9. Ni la loi organique (ordonnance $n^{\circ}$ 58-1067 du 7 novembre 1958 portant loi organique sur le Conseil constitutionnel) ni le règlement intérieur (Règlement intérieur sur la procédure suivie devant le Conseil constitutionnel pour les questions prioritaires de constitutionnalité) n'en font clairement état. C'est en fait la jurisprudence du Conseil constitutionnel elle-même qui est le plus explicite à cet égard (v. notamment $\mathrm{CC}, \mathrm{n}^{\circ}$ 2010-1 QPC, 28 mai 2010, Consorts L.).

10. Il semble aujourd'hui incontestable que "l'idée selon laquelle le Conseil constitutionnel serait exclusivement juge au fond de la contestation de constitutionnalité est contredite par la pratique "; Sénac C-É., L'office du juge constitutionnel. Etude du contrôle de constitutionnalité par les juridictions françaises, éd. L.G.D.J., coll. Bibliothèque Constitutionnelle et de Science politique, Tome 145, p. 156.

11. L'existence même de décisions de non-lieu à statuer accrédite l'existence d'un filtre de la recevabilité entre la décision de renvoi de la cour suprême et l'examen au fond de la question par le Conseil.

12. Douteaud S., «Pour une clarification de la distinction entre moyens inopérants et moyens irrecevables en contentieux administratif », loc. cit., p. 697.

13. En témoigne le fait qu'une QPC peut être jugée partiellement recevable, lorsque le Conseil estime avoir déjà partiellement répondu à le question et qu'aucun changement de circonstances n'est intervenu depuis, v. par ex. CC, n² 2016-598 QPC, 25 novembre 2016, Société Eurofrance.

14. CC, $n^{\circ}$ 2016-613 QPC, 24 février 2017, Département d'Ille-et-Vilaine, \$15. C'est une atteinte au principe d'égalité qui est en l'espèce invoquée ; v. également $C C, n^{\circ}$ 2015-498, 20 novembre 2015, Société SIACI Saint-Honoré SAS et autres, §3; CC, n² 2015-484 QPC, 22 septembre 2015, Société UBER France SAS et autre (II), \$17.

15. CC., $n^{\circ} 2018-698$ QPC, 6 avril 2018, Syndicat secondaire Le Signal \$11, c'est le droit de propriété qui est visé en l'espèce ; v. également CC, n²019-790 QPC, 14 juin 2019, Société ENR Grenelle Habitat et autres.

16. Comme le rappelle P-Y. Gahdoun, le non-lieu à statuer est fondé sur « un raisonnement autour de la recevabilité de la QPC, c'est-à-dire en statuant sur un élément qui rend la QPC admissible ou non d'un 
point de vue procédural », Gahdoun P-Y., «Les bienfaits de l'imperfection. Décision n 2019-790 QPC du 14 juin 2019 », Chroniques de droit public, in «Titre VII», 2019/2 N³, p. 127. Parallèlement, le juge constitutionnel peut conclure à la conformité alors même qu'au sens de la classification qu'il adopte pour les décisions d'irrecevabilité, il aurait dû rendre un non-lieu, cf. Benetti J., « Échec au renvoi : les décisions QPC de « non-lieu à statuer » ", in Revue du droit public, 2012, n 3, p. 593.

17. Pour une étude de cette question v. Hennette-Vauchez S. et Marguet L., «La QPC et les "droits et libertés que la Constitution garantit »: consécration et façonnage d'une nouvelle catégorie du droit constitutionnel », Dossier "Les 10 ans de la QPC ", in Revue des droits de l'homme, ce numéro.

18. S'agissant de la transmission de la question par les juridictions ordinaires au Conseil d'État ou à la Cour de cassation : Art. 23-1, al. 1 et 23-2, al.5 de l'ordonnance ${ }^{\circ}$ 58-1067 du 7 novembre 1958 portant loi organique sur le Conseil constitutionnel. S'agissant du renvoi de la question par le Conseil d'État ou la Cour de cassation au Conseil constitutionnel : Art. 23-5, al. 1 et al. 2 du même texte.

19. Art. 23-2, al. 1, 3. pour les juridictions ordinaires et 23-5 al. 3 pour le Conseil d'État et la Cour de cassation de l'ordonnance ${ }^{\circ}$ 58-1067 précitée.

20. V. Rousseau D., Gahdoun P-Y., Bonnet J., Droit du contentieux constitutionnel, éd. Montchrestien, Lextenso-éditions, 12ème éd., 2020, p. 237.

21. V. en ce sens, G. Toulemonde, I. Thumerel, D. Galati, «Les juridictions suprêmes renforcées dans leur office de cour suprême", in La QPC, le procès et ses juges. L'impact sur le procès et l'architecture juridictionnelle, E. Cartier (dir.), éd. Dalloz, 2013, Deuxième partie, 1, Section 2, 1., pp. 282-301. Ce pouvoir d'appréciation apparaît d'autant plus manifeste qu'il donne lieu à des divergences au sein des juridictions suprêmes et entre elles, v. Durand V. et Türk P., «Convergences et divergences autour de l'appréciation des caractères sérieux et/ou nouveau des QPC par les juridictions suprêmes", in La QPC, le procès et ses juges. L'impact sur le procès et l'architecture juridictionnelle, op. cit., Deuxième partie, 2, Section 1, Sous-section 2, pp. 486-495.

22. CE, 4 juillet 2014, Société FRP VII, n³75927, inédit; cité par C-E. Sénac, L'office du juge constitutionnel. Etude du contrôle de constitutionnalité par les juridictions françaises, op. cit., p. 205.

23. Cf. II pour les différents cas de figure.

24. De jurisprudence constante le Conseil constitutionnel se refuse d'ailleurs à apprécier l'applicabilité de la disposition contestée au litige (CC, n² 2010-1 QPC, 28 mai 2010, Consorts L., § 6) comme le caractère sérieux de la question transmise sauf si celle-ci lui est directement posée comme dans le cas du contentieux électoral (CC, n 2017-4977 QPC / AN, 7 août 2017, M. Raphaël Belaïche, §12).

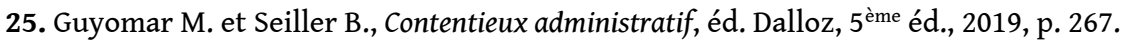

26. Guyomar M. et Seiller B., Contentieux administratif, op. cit., p. 262.

27. C-E. Sénac, L'office du juge constitutionnel. Etude du contrôle de constitutionnalité par les juridictions françaises, op. cit., p. 229.

28. V. par exemple CC, $\mathrm{n}^{\circ}$ 2012-252 QPC, 4 mai 2012, SELARL Le Discorde Deleau.

29. CC, $\mathrm{n}^{\circ}$ 2014-420/421 QPC, 9 octobre 2014, M. Maurice L.; CC, $\mathrm{n}^{\circ}$ 2013-349 QPC, 18 octobre 2013, Sociétés Allianz IARD et autre.

30. S'agissant par exemple de contrôler la constitutionnalité d'une disposition législative adoptée par référendum (CC, n 2014-392 QPC, 25 avril 2014, Province Sud de Nouvelle-Calédonie) ou d'une disposition qui « se borne à tirer les conséquences nécessaires de dispositions inconditionnelles et précises d'une directive de l'Union européenne » (CC, $\mathrm{n}^{\circ}$ 2010-79 QPC, 17 décembre 2010, M. Kamel D.).

31. L'expression est notamment employée dans les commentaires officiels pour désigner la recevabilité de la question dont le Conseil est saisi (Commentaire de la décision n 2019-790 QPC du 14 juin 2019, Société ENR Grenelle Habitat et autres, p. 7).

32. V. par ex. CC, $\mathrm{n}^{\circ}$ 2018-729 QPC, 7 septembre 2018, Société Tel and Com ; CC, $\mathrm{n}^{\circ}$ 2013-359 QPC, 13 décembre 2013, Société Sud Radio Services et autre; CC, n 2017-692 QPC, 16 février 2018, Époux F. 
33. Nous faisons ici référence à une très large part des décisions de non-lieu à statuer rendues par le Conseil constitutionnel.

34. Soit lorsque la question a été posée dans le cadre d'une procédure qui n'est pas une instance au sens de l'art. 61-1 C. (c'est notamment le cas de la procédure d'admission à l'aide juridictionnelle ; CC, $\mathrm{n}^{\circ}$ 2014-440 QPC, 21 novembre 2014, M. Jean-Louis M.), soit lorsque l'instance était éteinte au moment où la question est posée (n 2015-491 QPC, 14 octobre 2015, M. Pierre G.). On rappellera en revanche que «lorsque le Conseil a été saisi [...], l'extinction, pour quelque cause que ce soit, de l'instance à l'occasion de laquelle la question a été posée est sans conséquence sur l'examen de la question ", art. 23-9 de l'ordonnance n 58-1067 du 7 novembre 1958 portant loi organique sur le Conseil constitutionnel.

35. Soit les 730 décisions QPC rendues du 1er juin 2010, date des premières décisions, au 31 décembre 2019.

36. CC, $\mathrm{n}^{\circ}$ 2015-527 QPC, 22 décembre 2015, M. Cédric D. ; CC, nº 2017-624 QPC, 16 mars 2017, M. Sofiyan $I$.

37. Objectif de valeur constitutionnelle de lutte contre la fraude et l'évasion fiscales:CC, $\mathrm{n}^{\circ}$ 2018-735 QPC, 27 septembre 2018, M. Xavier B. et autres; objectif de valeur constitutionnelle d'intelligibilité et d'accessibilité de la loi : CC, 2012-283 QPC, 23 novembre 2012, M. Antoine de M. ; CC, $\mathrm{n}^{\circ}$ 2012-280 QPC, 12 octobre 2012, Société Groupe Canal Plus et autre.

38. Et ce depuis CC, $n^{\circ} 2010-5$ QPC, 18 juin 2010, SNC Kimberly Clark. C'est notamment le cas de la décision CC, $\mathrm{n}^{\circ}$ 2011-175 QPC, 7 octobre 2011, Société Travaux Industriels Maritimes et Terrestres et autres, alors même qu'est invoqué l'objectif de valeur constitutionnelle d'intelligibilité et d'accessibilité de la loi comme dans deux des décisions discutées (cf. note 36).

39. En l'occurrence la liberté d'entreprendre dans CC, $n^{\circ}$ 2018-735 QPC, précitée.

40. Si l'on s'en tient à la définition usuelle de l'exception comme "dérogation à la règle générale » (cf. le Dictionnaire Trésor de la langue française informatisé, entrée «Exception »), l'exception se trouve même dans une relation de dépendance univoque avec la règle puisque sans elle, il n'est point d'exception.

41. En témoignent la partition explicite entre le contrôle de la recevabilité et celui du fond ou encore le traitement expressément individué des différents griefs. Il ne fait pas non plus de doute que le Conseil tente, sans toujours y parvenir, de s'appuyer sur des formulations types qui, au gré de la jurisprudence et d'un usage sériel, constituent de véritables régimes jurisprudentiels.

42. On donne là encore l'impression de confondre recevabilité de la question et recevabilité des moyens mais il faut insister sur le fait que la nature objective du contentieux comme le contrôle abstrait opéré par le Conseil constitutionnel justifie largement cette confusion. L'appréciation sui generis de la recevabilité de la question constitue, comme on le verra, une part très marginale des décisions QPC ; dans la plupart des cas celle-ci est confondue avec la recevabilité de ses moyens.

43. Douteaud S., "Pour une clarification de la distinction entre moyens inopérants et moyens irrecevables en contentieux administratif ", loc. cit.

44. Ibid., p. 698.

45. Dans cette hypothèse seule l'irrecevabilité est contrôlée préalablement à l'examen du bienfondé, ibid., p. 701.

46. Dans cette hypothèse l'auteur de l'action peut agir sur la recevabilité d'un moyen (en respectant certaines conditions formelles et procédurales) tandis qu'il ne peut pas agir sur son opérance, ibid., p. 701.

47. Comme le rappelle S. Douteaud, à l'instar du contrôle de l'irrecevabilité, « il est acquis depuis plus de cinquante ans que l'opérance du moyen est elle aussi contrôlée préalablement à l'examen de son bien-fondé ", ibid.

48. Ibid., p. 705.

49. Ibid.

50. Ibid. 
51. Ibid., p. 706.

52. Nous souscrivons à l'idée que la tâche du commentateur peut supposer d'adopter des constructions conceptuelles distinctes de celles empruntées par le juge. V. Viala A., « Le point de vue du commentateur ", in Comment rédiger une décision de justice au XXIè siècle?, F. Malhière (dir.), éd. Dalloz, coll. Thèmes et commentaires, 2018, p. 102-103.

53. CC, $\mathrm{n}^{\circ}$ 2010-1 QPC, 28 mai 2010, Consorts L., \$6.

54. Art. 23-2, al. 1, 2., de l'ordonnance $n^{\circ}$ 58-1067 du 7 novembre 1958 portant loi organique sur le Conseil constitutionnel.

55. Ainsi logiquement d'une modification de la loi (CC, $\mathrm{n}^{\circ}$ 2015-513/514/526 QPC, 14 janvier 2016, M. Alain D. et autres) ou de la Constitution (CC, n 2012-233 QPC, 21 février 2012, Mme Marine Le Pen).

56. CC, $\mathrm{n}^{\circ}$ 2010-14/22 QPC, 30 juillet 2010, M. Daniel W. et autres, \$16-18.

57. Lorsqu'une disposition est mentionnée dans les motifs mais que «toutefois, le Conseil constitutionnel n'a pas déclaré cette disposition conforme à la Constitution dans le dispositif de sa décision", elle peut faire l'objet d'une question en l'absence même d'un changement de circonstances ; CC, $\mathrm{n}^{\circ}$ 2014-439 QPC, 23 janvier 2015, M. Ahmed S., §5. C'est donc la mention de la disposition dans le dispositif qui apparait déterminante.

58. CC, $\mathrm{n}^{\circ}$ 2013-331 QPC, 5 juillet 2013, Société Numéricâble SAS et autre, §8.

59. CC, $\mathrm{n}^{\circ}$ 2018-749 QPC, 30 novembre 2018, Société Interdis et autres.

60. Alexandre Palanco retient que le juge dispose d'une marge de manœuvre conséquente, pour ne pas dire totale, dans la reconnaissance d'un tel changement. Ainsi, ces "qualifications [...] peuvent être considérées comme erronées, discrétionnaires ou performatives selon les sensibilités", elles "sont au fondement d'une véritable instrumentalisation de la théorie du changement des circonstances", cf. Alexandre Palanco, «La théorie du changement des circonstances dans le contentieux de la QPC - Vers une évolution de l'office du Conseil constitutionnel ? ", in RFDA, 2019, pp. 328.

61. V. par exemple CC, $\mathrm{n}^{\circ} 2012-252 \mathrm{QPC}$, précitée.

62. CC, $\mathrm{n}^{\circ}$ 2014-420/421 QPC et CC, $\mathrm{n}^{\circ}$ 2013-349 QPC, précitées.

63. CC, $n^{\circ}$ 2011-120 QPC, 8 avril 2011, M. Ismaël A.

64. Rousseau D., Gahdoun P-Y., Bonnet J., Droit du contentieux constitutionnel, op. cit., p. 225 ; à propos de CC $n^{\circ}$ 2010-44 QPC, 29 septembre 2010, Epoux M., §9.

65. CC, $\mathrm{n}^{\circ}$ 2011-142/145 QPC, 30 juin 2011, Départements de la Seine-Saint-Denis et autres, \$17.

66. Pour reprendre les qualificatifs employés par P. Gervier. V. Gervier P., « Le changement de circonstances dans la jurisprudence du Conseil constitutionnel», in Revue du droit public, 2012, $\mathrm{n}^{\circ} 1, \mathrm{p} .89$ et $\mathrm{s}$.

67. CC, $\mathrm{n}^{\circ}$ 2017-681R QPC, 16 février 2018, Société Norbail-Immobilier; CC, $\mathrm{n}^{\circ}$ 2015-491R QPC, 11 décembre 2015, M. Pierre G.

68. CC, n² 2014-392 QPC, 25 avril 2014, Province Sud de Nouvelle-Calédonie ; en l'occurrence la loi du 9 novembre 1988 adoptée par référendum le 6 novembre 1988.

69. CC, n 62-20 DC, 6 novembre 1962, Loi relative à l'élection du Président de la République au suffrage universel direct.

70. CC, $\mathrm{n}^{\circ}$ 2010-79 QPC, 17 décembre 2010, M. Kamel D.

71. CC, $n^{\circ} 2006-540$ DC, 27 juillet 2006, Loi relative au droit d'auteur et aux droits voisins dans la société de l'information, $\$ 19$.

72. V. par ex. CC, $\mathrm{n}^{\circ}$ 2015-520 QPC, 3 février 2016, Société Metro Holding France SA venant aux droits de la société CRFP Cash, §9 ; CC, n² 2019-818 QPC, 6 décembre 2019, Mme Saisda C., §8.

73. Ce cas de figure ne s'est jamais présenté et de tels principes n'ont toujours pas reçu de définition jurisprudentielle subséquente. Pour une approche doctrinale, v. Viala A., « Le concept d'identité constitutionnelle : approche théorique ", in L'identité constitutionnelle saisie par les juges en Europe, Burgorgue-Larsen L. (dir.), éd. Pedone, 2011, pp. 7-26 ; Dubout E, «» Les règles ou 
principes inhérents à l'identité constitutionnelle de la France » : une supra-constitutionnalité ? ", in Revue française de droit constitutionnel, 2010, $\mathrm{n}^{\circ} 83$, pp. 451-482.

74. Douteaud S., «Pour une clarification de la distinction entre moyens inopérants et moyens irrecevables en contentieux administratif », loc. cit., p. 706.

75. CC, n² 2011-152 QPC, 22 juillet 2011, M. Claude C.

76. CC, $\mathrm{n}^{\circ}$ 2011-208 QPC,13 janvier 2012, Consorts B. Il en va de même des conventions collectives annexées à des lois, cf. Magnon X. (dir.), QPC. La question prioritaire de constitutionnalité. Principes généraux, pratique et droit du contentieux, Lexis-Nexis, 2ème éd., 2013, p. 76-77.

77. CC, n² 2011-219 QPC, 10 février 2012, M. Patrick É., \$3 ; CC, n 2014-412 QPC, 19 septembre 2014, M. Laurent D., §8.

78. Qu'il apparaît judicieux de mentionner quand bien même elle échapperait à notre corpus d'analyse ; CC, $\mathrm{n}^{\circ}$ 2020-843 QPC, 28 mai 2020, Force 5.

79. Sur cette question v. notamment, de Montecler M-C., «Ordonnances : duo ou duel au PalaisRoyal ? CE 1er juill. 2019, req. $\mathrm{n}^{\circ} 429132$; Cons. const. 3 juill. 2020, $\mathrm{n}^{\circ}$ 2020-851/852 QPC », in Dalloz Actualités, 9 juillet 2020 ; Carrère T., « La guerre des ordonnances aura-t-elle lieu ? A propos de la décision du Conseil constitutionnel n²020-843 QPC du 28 mai 2020 », in JP Blog, 8 juin 2020.

80. CC, $n^{\circ} 2013-334 / 335$ QPC, 26 juillet 2013, Société Somaf et autre.

81. Commentaire de la décision $n^{\circ} 2013-334 / 335$ QPC du 26 juillet 2013, p. 11.

82. Ibid., p. 10.

83. CC, $n^{\circ} 2019-790$ QPC, 14 juin 2019, Société ENR Grenelle Habitat et autres.

84. CC, $\mathrm{n}^{\circ}$ 2011-219 QPC, 10 février 2012, M. Patrick É., §5; CC, n 2015-460 QPC, 26 mars 2015, Comité de défense des travailleurs frontaliers du Haut-Rhin et autre.

85. CC, $n^{\circ}$ 2013-363 QPC du 31 janvier 2014, M. Michel P., §11.

86. On sait que la définition de cette catégorie est largement discrétionnaire et ne repose pas sur des critères objectifs (cf. V. Rousseau D., Gahdoun P-Y., Bonnet J., Droit du contentieux constitutionnel, op. cit., pp. 299-302). Elle emporte cependant des enjeux essentiels, v. HennetteVauchez S. et Marguet L., "La QPC et les «droits et libertés que la Constitution garantit »: consécration et façonnage d'une nouvelle catégorie du droit constitutionnel », Art. cit.

87. CC, $\mathrm{n}^{\circ}$ 2010-4/17 QPC, 22 juillet 2010, M. Alain C. et autre; CC, $\mathrm{n}^{\circ} 2012-241$ QPC, 4 mai 2012, EURL David Ramirez ; CC, $\mathrm{n}^{\circ}$ 2013-370 QPC, 28 février 2014, M. Marc S. et autre.

88. Commentaire de la décision $n^{\circ} 2015-471$ QPC du 29 mai 2015, p. 6, cité par Rousseau D., Gahdoun P-Y., Bonnet J., Droit du contentieux constitutionnel, op. cit., p. 303.

89. Ibid., p. 5.

90. On pense notamment aux objectifs de valeur constitutionnelle ou à l'incompétence négative du législateur (cf. II/B/).

91. Art. 14 DDHC ; CC, $\mathrm{n}^{\circ}$ 2010-19/27 QPC, 30 juillet 2010, Époux P. et autres, \$16.

92. Art. $72 \mathrm{C} ; \mathrm{CC}, \mathrm{n}^{\circ}$ 2013-304 QPC, 26 avril 2013, Commune de Maing, §7.

93. Qui découlerait de l'art. 3 C et 6 DDHC ; CC, n² 2015-471 QPC, 29 mai 2015, Mme Nathalie K.-M.

94. CC, $\mathrm{n}^{\circ}$ 2017-687 QPC, 2 février 2018, Association Wikimédia France et autre, \$15.

95. CC, n² 2019-785 QPC, 24 mai 2019, M. Mario S., \$6.

96. Commentaire de la décision $n^{\circ} 2014-440$ du 21 novembre 2014, p. 3. L'art. 23-7 prévoit en son alinéa 1 que dans le cas où la Cour de cassation ou le Conseil d'État ne se prononce pas, dans un délai de trois mois, sur la question qui lui est transmise, celle-ci est automatiquement renvoyée au Conseil constitutionnel.

97. Ibid.

98. CC, $n^{\circ}$ 2011-206 QPC, 16 décembre 2011, M. Noël C. ; CC, n² 2012-283 QPC, 23 novembre 2012, M. Antoine de M. ; CC, $\mathrm{n}^{\circ}$ 2013-363 QPC, 31 janvier 2014, M. Michel $P$.

99. Notre corpus en compte deux : $\mathrm{n}^{\circ}$ 2012-237 QPC, 15 février 2012, M. Zafer E. ; CC, n 2014-440 QPC, 21 novembre 2014, M. Jean-Louis $M$.

100. Cour de cassation, Chambre criminelle, 30 novembre 2011, 11-82.961, Inédit. 
101. CC, n²012-237 QPC, précité, §3-4.

102. On peut ainsi s'étonner que le Tribunal des Conflits n'en fasse pas partie, v. en ce sens, Nivert N., «Une réforme à faire? Plaidoyer pour l'extension de la question prioritaire de constitutionnalité devant le Tribunal des conflits », in RFDA, 2015, pp. 343-357.

103. Cf. alinéa 1 et 2 de l'article 23 de la loi du 10 juillet 1991.

104. Commentaire de la décision $n^{\circ} 2014-440$ du 21 novembre 2014, p. 5.

105. Douteaud S., «Pour une clarification de la distinction entre moyens inopérants et moyens irrecevables en contentieux administratif », loc. cit., p. 706.

106. Comme l'art. 6 de la Charte de l'environnement (CC, n² 2013-346 QPC, 11 octobre 2013, Société Schuepbach Energy LLC, \$19), le principe de péréquation financière de l'art. 72 C. (CC, $\mathrm{n}^{\circ}$ 2010-29/37 QPC, 22 septembre 2010, Commune de Besançon et autre, \$5) ou le principe de nationalisation pour cause de monopole (préambule de 1946, al. 9 ; CC, n 2015-459 QPC, 26 mars 2015, M. Frédéric P., \$6).

107. Quoique la terminologie adoptée par la haute instance ne suive pas toujours cette logique, ainsi le principe intégrant les langues régionales au patrimoine de la France est-il jugé « inopérant » alors qu'il semble en tout état de cause irrecevable (art. 75-1 C; CC, n 2011-130 QPC, 20 mai 2011, Mme Cécile L. et autres).

108. CC, $\mathrm{n}^{\circ} 2010-5$ QPC, 18 juin 2010, SNC Kimberly Clark.

109. CC, $\mathrm{n}^{\circ}$ 2013-364 QPC, 31 janvier 2014, Coopérative GIPHAR-SOGIPHAR et autre; CC, $\mathrm{n}^{\circ} 2013-367$ QPC, 14 février 2014, Consorts $L$.

110. S'agissant des modalités de recouvrement de l'imposition, le Conseil constitutionnel considère que la méconnaissance par le législateur de sa compétence n'affecte pas le droit de propriété, mais bien le droit à un recours effectif (CC, n 2014-419 QPC, 8 octobre 2014, Société Praxair SAS, §11).

111. CC, $\mathrm{n}^{\circ}$ 2010-4/17 QPC, 22 juillet 2010, M. Alain C. et autre, §9 ; CC, $\mathrm{n}^{\circ} 2011-175$ QPC, 7 octobre 2011, Société travaux industriels maritimes et terrestres et autres, §9. On notera que le moyen tiré de cet objectif peut être jugé opérant tout en emportant un examen au fond particulièrement lapidaire, v. CC, $\mathrm{n}^{\circ}$ 2013-370 QPC, 28 février 2014, M. Marc S. et autre, \$19.

112. CC, $\mathrm{n}^{\circ}$ 2014-422 QPC, 17 octobre 2014, Chambre syndicale des cochers chauffeurs CGT-taxis, $\$ 12$.

113. CC, $n^{\circ} 2018-735$ QPC, 27 septembre 2018, M. Xavier B. et autres, \$27.

114. CC, $\mathrm{n}^{\circ} 2014-434$ QPC, 5 décembre 2014, Société de laboratoires de biologie médicale Bio Dômes Unilabs SELAS, §7.

115. CC, $\mathrm{n}^{\circ}$ 2010-77 QPC, 10 décembre 2010, Mme Barta Z., \$3.

116. Une telle partition n'apparaît pas incontestable dès lors qu'on peut légitimement concevoir un lien intrinsèque entre certains objectifs de valeur constitutionnelle, comme celui d'intelligibilité et d'accessibilité de la loi ou celui de bonne administration de la justice, et la protection des droits et libertés fondamentales. Nous remercions Laurie Marguet d'avoir attiré notre attention sur ce point.

117. CC, $\mathrm{n}^{\circ}$ 2010-84 QPC, 13 janvier 2011, SNC Eiffage Construction Val de Seine, $\$ 4$; CC, $\mathrm{n}^{\circ} 2010-90$ QPC, 21 janvier 2011, M. Jean-Claude C., \$6 ; n²011-111 QPC, 25 mars 2011, Mme Selamet B., \$4; CC, $\mathrm{n}^{\circ}$ 2011-119 QPC, 1er avril 2011, Mme Denise R. et autre, §3 ; CC, $\mathrm{n}^{\circ}$ 2011-124 QPC, 29 avril 2011, Mme Catherine B., §3 ; CC, $\mathrm{n}^{\circ} 2015-487$ QPC, 7 octobre 2015, M. Patoarii R., §7 ; CC, n² 2016-598 QPC, 25 novembre 2016, Société Eurofrance, \$9.

118. CC, $\mathrm{n}^{\circ}$ 2012-233 QPC, 21 février 2012, Mme Marine Le Pen, §7.

119. CC, $\mathrm{n}^{\circ} 2013-326$ QPC, 5 juillet 2013, M. Jean-Louis M., $\$ 7$.

120. CC, $\mathrm{n}^{\circ}$ 2014-407 QPC, 18 juillet 2014, MM. Jean-Louis M. et Jacques B., §8.

121. Art. 1 et 3 de la Charte de l'environnement, CC, $n^{\circ} 2012-282$ QPC, 23 novembre 2012, Association France Nature Environnement et autre, §9 ; art. 7 de la Charte de l'environnement, CC, $\mathrm{n}^{\circ}$ 2014-411 QPC, 9 septembre 2014, Commune de Tarascon, $\$ 7$. 
122. C'est cependant le cas lorsque le Conseil constitutionnel considère que les dispositions relatives au mariage (CC, $\mathrm{n}^{\circ}$ 2010-92 QPC, 28 janvier 2011, Mme Corinne C. et autre, \$6) ou le droit de visite en droit de l'urbanisme (CC, n² 2015-464 QPC, 9 avril 2015, M. Marc A., §5) ne portent pas atteinte à la liberté individuelle.

123. D'autant qu'une telle audition peut donner lieu à une garde à vue, pour le cas où elle ferait naître une suspicion de commission d'infraction à l'encontre du témoin auditionné ; CC, $n^{\circ}$ 2011-191/194/195/196/197 QPC du 18 novembre 2011, Mme Élise A. et autres, \$16.

124. CC, $n^{\circ}$ 2010-12 QPC, 2 juillet 2010, Commune de Dunkerque, $\$ 5$.

125. En l'espèce le non-respect par les autorités administratives du principe de dignité de la personne humaine dans l'application des dispositions relatives à la garde à vue; CC, $\mathrm{n}^{\circ}$ 2010-14/22 QPC, 30 juillet 2010, M. Daniel W. et autres, \$20; v. également CC, $\mathrm{n}^{\circ}$ 2010-71 QPC, 26 novembre 2010, Mlle Danielle S., \$29; CC, n 2017-652 QPC, 4 août 2017, Comité d'entreprise de l'unité économique et sociale Markem Imaje, $\$ 11$.

126. On rappellera ici la difficulté à quantifier sur une notion dont les critères apparaissent opaques et indéterminés. Quine a pu considérer que «dire qu'une certaine quantification existentielle présuppose des objets d'une certaine espèce, c'est dire simplement que la formule ouverte qui suit le quantificateur est vraie de certains objets de cette espèce et d'aucun objet qui ne soit pas de cette espèce " (V. W. O. Quine, From a Logical Point of View, trad. fr., p. 186 ; cité par Boniface J., " Quine : indifférence de l'ontologie et réalisme", in Noesis [En ligne], 13|2008, mis en ligne le 15 décembre 2009, URL: http://journals.openedition.org/noesis/1617, §11). Inversement, ne pas pouvoir quantifier objectivement sur des objets démontre leur indétermination ou, à tout le moins, leur relativité ontologique.

127. D'aucuns considérant qu'elles sont, en pratique, les premiers destinataires de la décision QPC. On a pu considérer ainsi que «les simples particuliers apparaissent ainsi comme les éléments exogènes d'un contentieux dont ils altèrent la nature profonde», Thierry Santolini, "La question prioritaire de constitutionnalité au regard du droit comparé", in Revue française de droit constitutionnel, 2013/1 (nº 93), pp. 83 à 105, p. 101 ; cité par Benzina S., L'Effectivité des décisions QPC $d u$ Conseil constitutionnel, éd. L.G.D.J., coll. Bibliothèque constitutionnelle et de science politique, Tome 148, 2017, p. 637.

128. Girard D., "Le «non-lieu à statuer» devant le conseil constitutionnel : un élément révélateur de son ambivalence entre organe politique et cour constitutionnelle », loc. cit., e74 et e76.

129. P-Y. Gahdoun, « Les bienfaits de l'imperfection. Décision n² 2019-790 QPC du 14 juin 2019 », loc. cit., p. 123.

130. Girard D., «Le «non-lieu à statuer» devant le conseil constitutionnel : un élément révélateur de son ambivalence entre organe politique et cour constitutionnelle ", loc. cit., e64.

131. Les statistiques sont celles de 2017 (cf. Rapport du groupe de travail commun au Conseil d'État et à la Cour de cassation sur la question prioritaire de constitutionnalité, mai 2018, p. 2).

132. On a pu à cet égard proposer de réformer l'institution en lui conférant un «droit d'évocation " qui lui permettrait «d'attirer à lui les questions qui ne lui auraient pas été transmises et qu'il souhaiterait examiner", Rousseau D., Gahdoun P-Y., Bonnet J., Droit du contentieux constitutionnel, op. cit., p. 264.

133. Cf., Durand V. et Türk P., «Convergences et divergences autour de l'appréciation des caractères sérieux et/ou nouveau des QPC par les juridictions suprêmes ", in La QPC, le procès et ses juges. L'impact sur le procès et l'architecture juridictionnelle, loc. cit.

134. Pour justifier l'absence de prise en compte des situations de fait sur le contrôle de constitutionnalité de la disposition, le commentaire officiel se borne ainsi à rappeler qu' "en effet, comme le Conseil a eu l'occasion de le juger à plusieurs reprises, l'éventualité du non-respect de la loi, qu'il soit volontaire, dans le cas d'un détournement ou qu'il résulte d'un état de fait, ne saurait entacher la loi 
d'inconstitutionnalité", en faisant référence à la jurisprudence (cf. Commentaire de la décision $n^{\circ}$ 2017-652 QPC du 4 août 2017, p. 14).

135. En effet, les moyens écartés par le considérant dit «balai » peuvent en théorie l'être pour des questions d'irrecevabilité ou d'inopérance sans que cela n'apparaisse expressément. V. notamment, Sénac C-É., L'office du juge constitutionnel. Etude du contrôle de constitutionnalité par les juridictions françaises, op. cit., p. 435-438.

136. Malhière F., La brièveté des décisions de justice (Conseil constitutionnel, Conseil d'État, Cour de cassation), éd. Dalloz, coll. Nouvelle Bibliothèque des Thèses, 2013.

137. V. en ce sens Maestracci N. et Cassard-Valembois A-L., «Le nouveau mode de rédaction des décisions du Conseil constitutionnel ", in Comment rédiger une décision de justice au XXIè siècle ?, éd. Dalloz, coll. Thèmes et commentaires, 2018, pp. 13-38.

138. Gahdoun P-Y., « Les bienfaits de l'imperfection. Décision n² 2019-790 QPC du 14 juin 2019 », loc. cit.

\section{RÉSUMÉS}

L'étude de dix ans de décisions QPC rendues par le Conseil constitutionnel est l'occasion d'apprécier la manière dont ce dernier en a tiré profit pour structurer sa jurisprudence et les catégories qui s'y expriment. Nous nous intéressons plus précisément au rôle de troisième filtre que s'arroge dans ce cadre le Conseil constitutionnel. En effet, si les juridictions du filtre appliquent ouvertement des critères leur permettant d'apprécier l'opportunité de renvoyer une question prioritaire de constitutionnalité au Conseil constitutionnel, ce dernier examine également, quoique moins explicitement, la question de la validité de sa saisine. Un tel contrôle le conduit ponctuellement à ne pas répondre à la question posée en relevant qu'elle est fondée sur des moyens irrecevables ou des moyens inopérants. Notre contribution s'attache à éclairer ces catégories autant qu'à étudier les motivations qui s'y rattachent dans la jurisprudence relative à la question prioritaire de constitutionnalité. Elle donne à voir les stratégies discursives mise en œuvre par le Conseil constitutionnel pour éviter de répondre à la question qui lui est posée, tout en dessinant les enjeux qu'implique un tel refus pour les différents acteurs de cette procédure.

The study of ten years of preliminary ruling on constitutionality tried by the Constitutional Council is an opportunity to appreciate the way in which this court has taken advantage of them to structure its jurisprudence and its categories. We are specifically focused on the role of third filter that the Constitutional Council has assumed in this context. Indeed, although the filter courts openly apply criteria that enable them to assess the appropriateness of referring a preliminary ruling on constitutionality to the Constitutional Council, the latter also examines, albeit less explicitly, the question of the validity of this referral. Such a control occasionally leads to not answer the question by noting that it is based on inadmissible or inoperative pleas. Our contribution seeks to shed light on these categories as well as to study the motivations that are linked to them in the case-law related to the preliminary ruling on constitutionality. It shows the discursive strategies used by the Constitutional Council to avoid answering the question, while at the same time outlining the stakes that such a refusal implies for the various actors in this procedure. 
INDEX

Mots-clés : question prioritaire de constitutionnalité, juridiction du filtre, moyen inopérant, moyen irrecevable

Keywords : preliminary ruling on constitutionality, filter courts, inoperative plea, inadmissible plea.

\section{AUTEUR}

THOMAS ACAR

Thomas Acar est Docteur en droit public, enseignant-chercheur contractuel à l'Université de Cergy-Pontoise, CPJP et chercheur rattaché au CTAD (équipe CREDOF) 